\title{
Variación temporal en la dieta del congrio colorado, Genypterus chilensis (Guichenot, 1881) frente al litoral de Talcahuano, Chile (36 $32^{\prime} \mathrm{S}$ - $36^{\circ} 45^{\prime}$ 'S)
}

\author{
Temporal variation in the diet of the red ling, Genypterus chilensis (Guichenot, 1881) \\ in the coast off Talcahuano, Chile (36 $32^{\prime}$ 'S - 36 $45^{\circ} \mathrm{S}$ ) \\ Javier Chong ${ }^{1}$, Karina Sepúlveda ${ }^{1}$ y Christian M. Ibáñez ${ }^{2}$ \\ ${ }^{1}$ Departamento de Ecología Costera, Facultad de Ciencias, Universidad Católica de la Santísima Concepción, Casilla 297, \\ Concepción, Chile \\ ${ }^{2}$ Departamento de Ciencias Ecológicas, Facultad de Ciencias, Universidad de Chile, Casilla 653, Santiago, Chile \\ jvchong@ucsc.cl
}

\begin{abstract}
A study of the gastric content of G. chilensis carried out in the area of Talcahuano is presented, based on samples obtained during two periods: 1974-75 and 1996-97. In the second period, monthly samples were obtained with the objective of performing seasonal comparisons in the diet. The diet was described by means of frequency, numeric and gravimetric methods, and numeric and relative importance index. Diversity was compared among years of sampling and between seasons of the second period. The red ling possesses a trophy spectrum with a clear preference toward the euriphagy in the study area, since it feeds at least of 38 types of prey corresponding to crustaceans, mollusks and fish, with Pleuroncodes monodon dominant in both periods. Differences in richness and diversity of prey among years were recorded; furthermore there were differences between spring and winter in the period 1996-97. The high consumption of benthic crustaceans, particularly of $P$. monodon, could be attributed to a higher consumption of more abundant prey, and the feeding preference would be associated with its way of benthic life and the environmental offer in the area and study time. The variations in the diet between years and seasons would depend on the changes in composition, abundance and diversity of the preys in the study area.
\end{abstract}

Key words: Genypterus chilensis, feeding, temporal variations, prey diversity
Resumen.- Se presenta un estudio del contenido gástrico de G. chilensis realizado en la zona de Talcahuano, sobre la base de muestras obtenidas durante dos periodos: 1974-75 y 1996-97. En el segundo periodo se obtuvieron muestras mensuales para realizar comparaciones estacionales en la alimentación. La dieta se describió mediante los métodos de frecuencia de ocurrencia, numérico y gravimétrico, y los índices de importancia numérica y relativa. Se comparó la diversidad de la dieta entre años de muestreo y entre estaciones en el segundo periodo. Se determinó que el congrio colorado posee un espectro trófico con una clara tendencia hacia la eurifagia en el área de estudio, ya que se alimenta al menos de 38 tipos de presas correspondiente a crustáceos, moluscos y peces, siendo preponderante en ambos periodos Pleuroncodes monodon. Se encontraron diferencias en riqueza y diversidad de presas entre años y, en el periodo 1996-97 se registraron diferencias en la alimentación de primavera con respecto a la de invierno. El alto consumo de crustáceos y en particular de $P$. monodon se debería principalmente a que el congrio colorado utilizaría presas de mayor abundancia, cuya preferencia alimentaria estaría asociada con su modo de vida bentónica y la oferta ambiental en la zona y época de estudio. Las variaciones en la dieta entre años y estaciones dependen de los cambios en composición, abundancia y diversidad de las presas en el área de estudio.

Palabras clave: Genypterus chilensis, alimentación, variación estacional, diversidad de presas 


\section{Introducción}

Los congrios (Actinopterygii: Ophidiidae) constituyen en Chile recursos muy importantes en la pesca artesanal, particularmente el congrio colorado; en cambio el congrio negro y congrio dorado han ido aumentando cada vez mas su importancia en las capturas industriales, especialmente en la pesquería sur-austral (SERNAPESCA, 2004). En la década de los 80 se hacía mención de que a pesar de ser recursos importantes en la pesquería artesanal chilena, eran escasos los antecedentes biológicos sobre las tres especies de congrio: Genypterus blacodes (Schneider), G. chilensis (Guichenot) y G. maculatus (Tschudi) (Bahamonde \& Zavala 1981, George-Nascimento \& Huet 1984), situación que en parte ha tratado de ser solucionada con un proyecto del Fondo de Investigación Pesquera (FIP 2001-15), cuyos objetivos principales dicen relación con la biología reproductiva y la estructura de edad y crecimiento de las tres especies. En Chile estos peces habitan sobre la plataforma continental desde el submareal somero hasta los $400 \mathrm{~m}$ de profundidad en donde se alimentan principalmente de crustáceos y peces (Mann 1954, Bahamonde \& Zavala 1981, Henríquez \& Bahamonde 1964).

Los congrios colorado, G. chilensis, y negro, G. maculatus se encuentran sólo en la costa sudamericana del Pacífico sureste, desde Perú al extremo sur de Chile (Chirichigno 1974, Avilés 1979). En cambio, G. blacodes es una especie que tiene una distribución geográfica más amplia abarcando las costa del sur de Sudamérica, Australia y Nueva Zelanda (Mitchell 1984), y por lo tanto ha sido más estudiada. En Chile, las tres especies se alimentan de crustáceos entre los cuales destacan Pleuroncodes monodon y Pterygosquilla armata (Mann 1954, Henríquez \& Bahamonde 1964, Bahamonde \& Zavala 1981, Ruiz 1995). Los estudios de la dieta de estas especies en Chile son escasos y sólo descriptivos, salvo para el caso de G. maculatus donde existen antecedentes mas completos (Henríquez \& Bahamonde 1964, Bahamonde \& Zavala 1981). En cambio, los estudios de la dieta de G. blacodes que se han realizado en Nueva Zelanda y en las Islas Malvinas, demuestran que los hábitos alimentarios cambian estacionalmente, y que tienen relación con el tamaño corporal, ya que los ejemplares pequeños consumen principalmente crustáceos y los mayores preferentemente peces (Mitchell 1984, Nyegaard et al. 2004).
De las tres especies, la menos estudiada es $G$. chilensis y los insuficientes antecedentes dicen relación principalmente con su taxonomía (Norman 1937, Chocair et al. 1969, Pequeño 1989), distribución batimétrica y localización de la pesquería (Avilés 1979), su fauna parasitaria (Vergara \& George-Nascimento 1982, George-Nascimento et al. 2004) y con aspectos básicos sobre su biología reproductiva, edad y crecimiento y estructura de talla de las capturas en el litoral de la IV a la VIII Región (Tascheri et al. 2003).

En Chile, durante 1994, se observó el mayor desembarque de congrio colorado, $1.712 \mathrm{t}$, donde la mayor cantidad de las capturas se registraron en la zona centro-sur (Regiones V a IX) (SERNAPESCA 2004). El volumen de los desembarques ha ido en disminución y el registro más bajo en las últimas décadas corresponde a $100 \mathrm{t}$ el año 2003 (SERNAPESCA 2004). Este descenso seguramente es en parte reflejo de la carencia de antecedentes sobre esta especie, a lo que se suma la poca atención de los organismos encargados de la administración pesquera, debido a la ausencia de medidas de regulación sobre este recurso.

El presente estudio tiene por objetivo aportar al conocimiento de la ecología trófica de esta especie en el ecosistema nerítico de la costa frente a Chile central, mediante una comparación de antecedentes temporales de la dieta en una escala estacional y entre años.

\section{Material y métodos}

Se analizó un total de 1165 ejemplares de congrio colorado, de los cuales 469 correspondieron al periodo 1974-75 y 696 ejemplares al periodo 1996-97, que fueron obtenidos mediante espinel por embarcaciones artesanales que operaron en la zona costera comprendida entre caleta Cocholgüe y bahía San Vicente $\left(36^{\circ} 32^{\prime} \mathrm{S}-36^{\circ} 45^{\prime} \mathrm{S}\right)$. En todos los muestreos, a cada ejemplar se le midió la longitud total $(\mathrm{cm})$, se registró el peso $(10 \mathrm{~g})$ y se le extrajo el estómago, los que fueron congelados, para su posterior examen.

Los estómagos se analizaron individualmente y los contenidos fueron separados para el recuento y pesaje de cada presa; el registro de peso no se realizó durante el periodo 1974-75. La identificación de las presas se efectuó sobre la base de la literatura de Retamal (1981) para los crustáceos, Chirichigno (1974) y Ruiz (1995) en el caso de los peces y Nesis (1987) para los cefalópodos. El nivel taxonómico determinado estuvo 
condicionado por el grado de digestión que presentó el contenido estomacal.

La importancia relativa de cada presa en el contenido gástrico fue determinada utilizando los métodos cuantitativos básicos, expresados en porcentajes (Hyslop 1980): método numérico (\% N), método gravimétrico ( $\% \mathrm{G})$, y método de frecuencia de ocurrencia $(\% \mathrm{~F})$.

Posteriormente, para comparar la dieta entre años y considerando la ausencia de registro del peso de los contenidos en el periodo 1973-74, se utilizó el índice de importancia numérica (INN) (Windell 1968), expresado porcentualmente.

$$
\mathrm{IIN}=(\% \mathrm{~N}+\% \mathrm{~F})^{1 / 2} \times 100
$$

En cambio, para comparar la dieta entre estaciones durante el periodo 1996-97 se usó el índice de importancia relativa (IIR) (Pinkas et al. 1971).

$$
\mathrm{IIR}=(\% \mathrm{~N}+\% \mathrm{G}) \times \% \mathrm{~F}
$$

La comparación de las dietas entre años y entre estaciones para el segundo periodo de estudio se realizó mediante el índice de similitud porcentual (ISP) (Whittaker 1952 fide Hallacher \& Roberts 1985) que puede utilizar como base los índices de importancia numérica y relativa.

$$
I S P=100 * \sum_{i=1}^{S} \min (a i, b i)
$$

Donde: $a_{i} y b_{i}$ representan el porcentaje de IIR de la iésima categoría de presa en la dieta y la $\mathbf{s}$ corresponde al número total de categorías de presas comunes en las dietas que se comparan.

Además, para comparar entre estaciones del periodo 1996-97 se utilizó también el índice de similitud de Bray-Curtis (Ludwig \& Reynolds 1988) de acuerdo a la siguiente fórmula:

$$
\text { Bray-Curtis: }=\Sigma\left|\mathrm{X}_{\mathrm{ij}}-\mathrm{X}_{\mathrm{ik}}\right| / \Sigma\left(\mathrm{X}_{\mathrm{ij}}+\mathrm{X}_{\mathrm{ik}}\right)
$$

Como técnica de ordenación dietaria se utilizó la estrategia aglomerativa jerárquica de la media no ponderada (UPGMA) (Ludwig \& Reynolds 1988, Vivanco 1999). En este método, la distancia entre dos agrupaciones es calculada como la distancia promedio entre todos los pares de objetos en las dos diferentes agrupaciones (Ludwig \& Reynolds 1988, Vivanco 1999). Finalmente, se realizaron 1000 réplicas de bootstrap con el objetivo de comprobar si las agrupaciones entre estaciones son consistentes en similitud dietaria. El porcentaje de réplicas que soporta cada nodo se representan en el dendrograma de la abundancia de presas (Bray-Curtis). Además, se compararon los valores de IIR\% entre presas y estaciones mediante la prueba no-paramétrica de Friedman (Zar 1984). Todos los análisis se realizaron en el programa PAST.exe (Hammer et al. 2001).

En la comparación entre periodos (1974-75 y 199697) y estaciones del año 1996-97 se calculó el índice de diversidad de Shannon-Wienner y la dominancia de Simpson (Ludwig \& Reynolds 1988) basándose en los datos de abundancias de presas. Se realizó una comparación pareada de la diversidad de ShannonWienner usando una prueba de t-Student (Hammmer et al. 2001) y para obtener la varianza y el promedio de la diversidad de Shannon-Wienner se efectuó un boostrap que generó 1000 matrices aleatorias para los pares comparados (años y estaciones), manteniendo en cada fila y columna el mismo total de la matriz original.

\section{Resultados}

Durante el periodo 1974-75 se examinaron 469 ejemplares de congrio colorado de los cuales 200 presentaron contenido gástrico y sus tallas (LT) fluctuaron entre 31 y $115 \mathrm{~cm}$ (Fig. 1). Por su parte, en el periodo 1996-97 se examinaron 696 ejemplares, pero solamente 193 tenían contenido gástrico; las tallas estuvieron comprendidas entre 21 y $105 \mathrm{~cm}$, y pesos entre 1.000 y $10.000 \mathrm{~g}$ (Fig. 1).

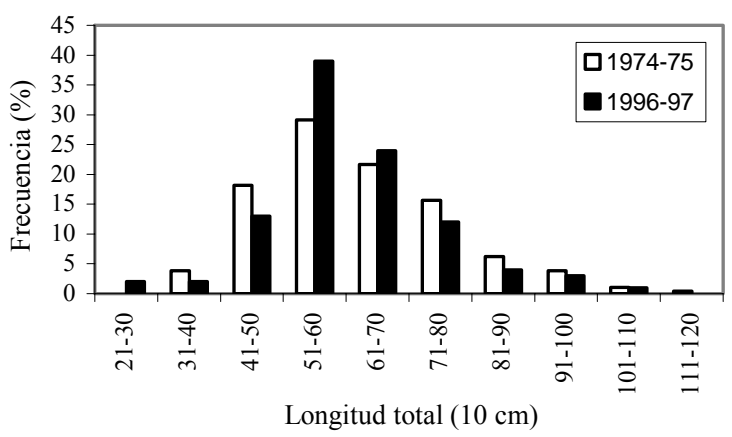

Figura 1

\section{Distribución de frecuencia por rango de tallas de G. chilensis}

Frequency distribution for length range of $G$. chilensis 
La dieta de $G$. chilensis en el periodo 1974-75 estuvo constituida principalmente por 20 especies de crustáceos, siendo Pleuroncodes monodon la presa más importante ( $\mathrm{IIN}=15,65 \%)$, secundariamente por cuatro especies de peces y dos de moluscos bentónicos (Tabla 1). En este periodo, el $42,6 \%$ de los estómagos presentó contenido gástrico. En cambio, durante el periodo 199697, la dieta estuvo constituida por 10 especies de crustáceos bentónicos, manteniéndose $P$. monodon como la presa más importante $(\mathrm{IIN}=21,45 \%), 12$ especies de peces y un calamar, pero sólo el $27,7 \%$ de los estómagos contenía alimento (Tabla 1).

Tabla 1

Porcentaje en número (\%N), frecuencia de ocurrencia (\%F) e índice de importancia numérica de los items presa presentes en los estómagos de G. chilensis, obtenidas en 1974-1975 $(n=200)$ y 1996-1997 $(n=193)$

Percentage (N\%), frequency (F\%) and numeric importance index of prey items in the stomachs of G. chilensis, obtained in 1974-1975 $(\mathrm{n}=200)$ and 1996-1997 $(\mathrm{n}=193)$

\begin{tabular}{|c|c|c|c|c|c|c|}
\hline \multirow[b]{2}{*}{ Presas } & \multicolumn{3}{|c|}{$1974-75$} & \multicolumn{3}{|c|}{$1996-97$} \\
\hline & $\% \mathrm{~N}$ & $\% \mathrm{~F}$ & $\%$ IIN & $\% \mathrm{~N}$ & $\% \mathrm{~F}$ & $\%$ IIN \\
\hline \multicolumn{7}{|l|}{ MOLLUSCA } \\
\hline Pelecypoda & 0,53 & 3,41 & 3,44 & & & \\
\hline Nassarius gayi & 2,94 & 9,85 & 6,19 & & & \\
\hline Loligo gahi & & & & 0,05 & 0,52 & 1,55 \\
\hline \multicolumn{7}{|l|}{ CRUSTACEA } \\
\hline Pleuroncodes monodon & 68,14 & 13,64 & 15,65 & 84,77 & 24,05 & 21,45 \\
\hline Emerita analoga & 0,18 & 0,38 & 1,30 & & & \\
\hline Petrolisthes desmarestii & 0,29 & 1,89 & 2,56 & 2,81 & 19,39 & 9,69 \\
\hline Cancer porteri & 7,54 & 15,15 & 8,24 & 1,43 & 4,66 & 5,07 \\
\hline Cancer plebejus & 0,65 & 2,65 & 3,14 & & & \\
\hline Cancer edwarsi & 0,06 & 0,38 & 1,15 & 0,05 & 0,03 & 0,58 \\
\hline Cancer coronatus & 0,18 & 1,14 & 1,99 & 0,05 & 0,52 & 1,55 \\
\hline Cancer setosus & & & & 0,05 & 0,52 & 1,55 \\
\hline Homalaspis plana & 0,06 & 0,38 & 1,15 & & & \\
\hline Murcia gaudichaudi & 0,47 & 1,89 & 2,66 & 3,24 & 9,84 & 7,44 \\
\hline Cervimunida johni & & & & 1,23 & 4,15 & 4,77 \\
\hline Allopetrolisthes sp. & & & & 0,10 & 0,52 & 1,62 \\
\hline Pterygosquilla armata & 0,24 & 1,52 & 2,30 & 0,41 & 2,60 & 3,57 \\
\hline Pseudocorystes sicarius & 0,12 & 0,76 & 1,62 & & & \\
\hline Alpheus chilensis & 1,00 & 4,55 & 4,08 & & & \\
\hline Austropandalus grayi & 1,77 & 1,89 & 3,31 & & & \\
\hline Betaeus truncatus & 1,41 & 1,89 & 3,14 & & & \\
\hline Heterocarpus reedi & 7,66 & 9,09 & 7,08 & & & \\
\hline Nauticaris magellanica & 0,06 & 0,38 & 1,15 & & & \\
\hline Rhynchocinetes typus & 0,12 & 0,76 & 1,62 & & & \\
\hline Euphausiacea & 0,24 & 0,76 & 1,73 & & & \\
\hline Serolis sp. & 0,12 & 0,76 & 1,62 & & & \\
\hline Pterygosquilla armata & 0,24 & 1,52 & 2,30 & & & \\
\hline Restos de crustáceos & 1,41 & 9,09 & 5,61 & 1,07 & 4,41 & 4,81 \\
\hline \multicolumn{7}{|l|}{ CHONDRICHTHYES } \\
\hline Schroederichthys chilensis & & & & 0,05 & 0,52 & 1,55 \\
\hline Raja sp. & & & & 0,05 & 0,52 & 1,55 \\
\hline \multicolumn{7}{|l|}{ OSTEICHTHYES } \\
\hline Engraulis ringens & & & & 0,21 & 1,04 & 2,30 \\
\hline Strangomera bentincki & 0,53 & 1,89 & 2,69 & 0,15 & 1,04 & 2,24 \\
\hline Genypterus sp. & 0,35 & 2,27 & 2,80 & 0,05 & 0,52 & 1,55 \\
\hline Normanichthys crockeri & 2,18 & 3,03 & 3,95 & 1,79 & 5,70 & 5,63 \\
\hline Prolatilus jugularis & 0,24 & 1,52 & 2,30 & 0,77 & 6,74 & 5,64 \\
\hline Helicolenus lengerichi & & & & 0,05 & 0,52 & 1,55 \\
\hline Merluccius gayi & & & & 0,15 & 1,04 & 2,24 \\
\hline Hippoglossina macrops & & & & 0,05 & 0,52 & 1,55 \\
\hline Sicyases sanguineus & & & & 0,05 & 0,52 & 1,55 \\
\hline Clinidae gen sp. & & & & 0,36 & 0,52 & 1,93 \\
\hline Peces indeterminados & 1,24 & 7,95 & 5,25 & 1,20 & 10,54 & 7,05 \\
\hline
\end{tabular}


Tabla 2

Índice de importancia relativa (IIR\%) para los principales ítems presa presentes en la dieta de G. chilensis, obtenidas durante las estaciones de 1996-1997

Index of relative importance (IRI\%) of principal prey items in the diet of G. chilensis obtained during the 1996-1997 seasons

\begin{tabular}{|c|c|c|c|c|}
\hline Presas & Otoño & Invierno & Primavera & Verano \\
\hline Pleuroncodes monodon & 1,05 & 85,04 & 70,90 & 81,18 \\
\hline Cervimunida johni & 17,39 & & 0,09 & \\
\hline Petrolisthes desmarestii & 2,15 & 10,50 & 11,12 & 0,24 \\
\hline Allopetrolisthes sp. & & & 0,02 & \\
\hline Murcia gaudichaudi & & 1,60 & 2,68 & 6,33 \\
\hline Cancer edwardsi & 0,16 & & & \\
\hline Cancer coronatus & & & & 0,05 \\
\hline Cancer setosus & & 1,04 & & \\
\hline Cancer porteri & 1,22 & 0,42 & 0,24 & \\
\hline Pterygosquilla armata & & & 0,04 & 0,75 \\
\hline Restos de crustáceos & 1,53 & & 1,41 & 0,02 \\
\hline Loligo gahi & & & 0,02 & \\
\hline Raja sp. & & & 0,42 & \\
\hline Schroederichthys chilensis & & & 0,05 & \\
\hline Engraulis ringens & & 0,14 & 1,23 & \\
\hline Normanichthys crockeri & 73,59 & & 0,05 & \\
\hline Prolatilus jugularis & & & 9,35 & 8,51 \\
\hline Clinidae gen. sp. & & & 0,02 & \\
\hline Helicolenus lengerichi & & & 0,03 & \\
\hline Strangomera bentincki & & & & 0,25 \\
\hline Genypterus sp. & & & 0,17 & \\
\hline Hippoglossina macrops & & & 0,02 & \\
\hline Sicyases sanguineus & & & 0,03 & \\
\hline Merluccius gayi & & 0,28 & & 0,33 \\
\hline Restos de peces & 2,91 & 0,98 & 2,11 & 2,34 \\
\hline
\end{tabular}

En el periodo 1974-75 la dieta de G. chilensis presentó una mayor riqueza (28 especies) y diversidad $(1,27)$ en relación con 1996-97 (25 y 0,67, respectivamente), por lo cual, la diversidad de la dieta resultó distinta entre años $(\mathrm{t}=2,58, \mathrm{P}=0,01)$.

En relación con la dieta estacional de G. chilensis durante el periodo 1996-97, en otoño, la presa más importante fue el pez Normanichthys crockeri (IIR = $73,59 \%$ ) mientras que en invierno, primavera y verano lo fue Pleuroncodes monodon (IIR $=85,04 \%, 70,9 \% \mathrm{y}$ $81,18 \%$, respectivamente) (Tabla 2). Debido a esto, los valores de IIR\% de las presas resultaron distintos entre estaciones (Friedman: $\chi^{2}=11,17$, g.l. $=3, P=0,010$ ). Esto es corroborado por los valores del índice de BrayCurtis, ya que otoño representa un grupo distinto de las otras estaciones que comparten más de un $80 \%$ de similitud, siendo soportada esta fuerte asociación por los valores de bootstrap $>95 \%$ (Fig. 2). Este resultado también es respaldado por el índice de similitud porcentual (ISP) que muestra una sobreposición de dieta $>82 \%$ entre las estaciones de invierno, primavera $\mathrm{y}$ verano y una semejanza $<6 \%$ con el otoño (Tabla 3 ).

\section{Tabla 3}

Valores del índice de similitud porcentual entre estaciones durante 1996-1997

Values of the similarity percentage Index between the seasons of 1996-1997

\begin{tabular}{lccc}
\hline & Invierno & Primavera & Verano \\
\hline Otoño & 4,60 & 5,78 & 3,65 \\
Invierno & & 84,36 & 82,68 \\
Primavera & & & 84,73 \\
\hline
\end{tabular}


La diversidad se mantuvo alta en todas las estaciones siendo primavera la que tuvo los valores más altos y el invierno los más bajos, mientras que la dominancia se mantuvo constante con valores entre 0,5 a 0,7 y que indican que la dominancia es compartida entre especies (Fig. 3); sólo se encontraron diferencias significativas en la diversidad entre las estaciones de invierno y primavera (Tabla 4).

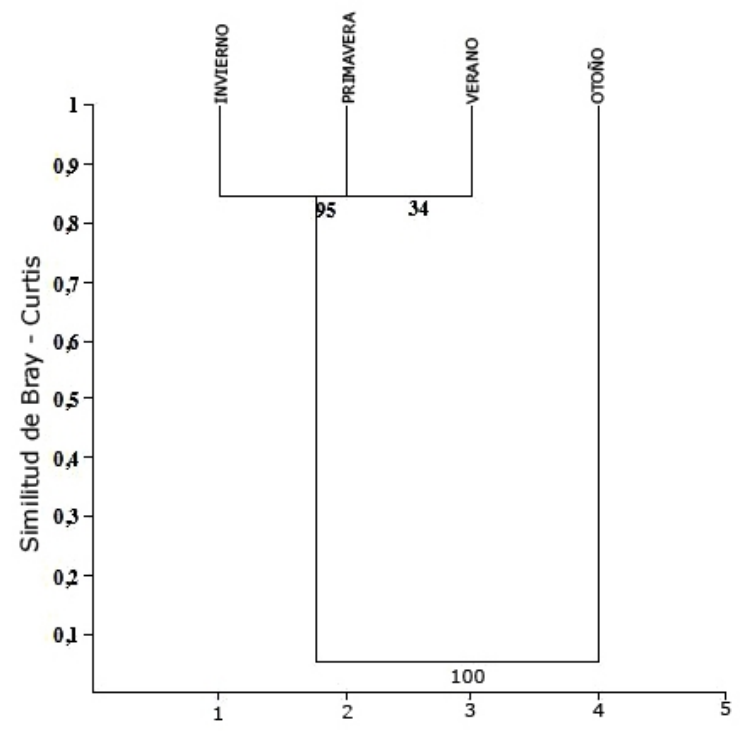

Figura 2

Dendrograma de la similitud (Bray-Curtis) dietaria de $G$. chilensis entre las estaciones de 1996-1997

Dendogram of diet similarity (Bray-Curtis) of G. chilensis among seasons from 1996-1997

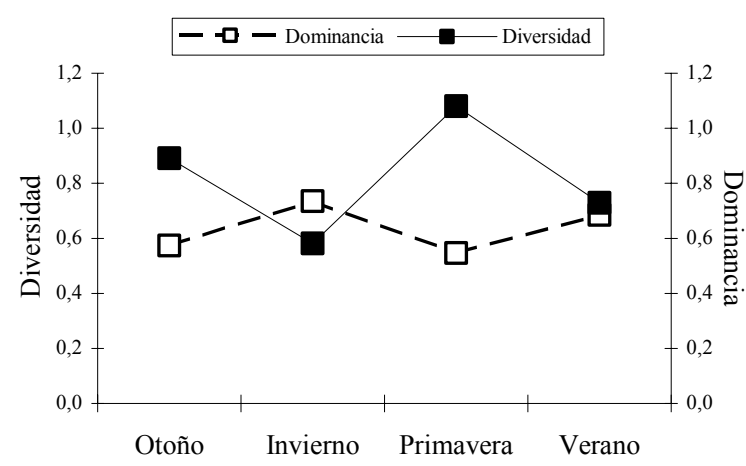

Figura 3

Diversidad y dominancia de la dieta de G. chilensis entre las estaciones de 1996-1997

Diversity and dominance of the diet of G. chilensis among seasons from 1996-1997
Tabla 4

Valores del estadígrafo t-Student de las comparaciones de la diversidad de Shannon-Wienner entre estaciones en el periodo 1996-1997

Statistics of t-Student values of the comparisons of Shannon-Wienner diversity among seasons of 1996-1997

\begin{tabular}{lccc}
\hline & Invierno & Primavera & Verano \\
\hline Otoño & 1,9371 & $-0,7242$ & 1,0462 \\
Invierno & & $-2,5111^{* *}$ & 0,8417 \\
Primavera & & & 1,6786 \\
$* * P<0,001$ & & &
\end{tabular}

\section{Discusión}

La dieta de G. chilensis en el periodo 1974-75 estuvo constituida principalmente por 20 especies de crustáceos y 4 de peces, mientras que en periodo 19961997 estuvo constituida por 10 especies de crustáceos y 12 de peces. En general, la dieta de G. chilensis es muy similar a lo informado para G. maculatus en Chile (Henríquez \& Bahamonde 1964, Bahamonde \& Zavala 1981) y para G. blacodes de Chile (Bahamonde \& Zavala 1981), de Nueva Zelanda (Mitchell 1984) y de las Islas Malvinas (Nyegaard et al. 2004).

La gran cantidad de estómagos vacíos (57,4\% y $72,3 \%$ ) se debería al cambio de presión al momento de la captura que provoca una gran dilatación de la vejiga gaseosa lo que provoca una expulsión de los alimentos ingeridos al ser izado rápidamente a la embarcación (Henríquez \& Bahamonde 1964).

La composición así como la diversidad de la dieta de G. chilensis resultó distinta entre años; esto debido a que en el periodo 1974-75 se alimentaron de una mayor cantidad de especies de crustáceos y en cambio en el periodo 1996-97 se alimentaron de una mayor cantidad de especies de peces, no obstante en ambos periodos la presa más importante fue $P$. monodon, un langostino relativamente abundante en la zona de estudio. Bahamonde \& Zavala (1981) sugieren que las variaciones entre años en la dieta de $G$. maculatus se deberían a cambios en la composición y abundancia de las presas. En este estudio el número de muestras y el lugar de estudio son similares por lo tanto los cambios en la dieta entre años estarían influenciados por la composición, abundancia y diversidad de las presas en la zona de Talcahuano. 
En el periodo 1996-97 la composición de la dieta presentó cambios estacionales, siendo $P$. monodon la presa más importante en invierno, primavera y verano, exceptuando el otoño en donde lo fue Normanichthys crockeri, especie que tiende a ser menos abundante durante el invierno (Tabla 2, Fig. 1). Esto es semejante a lo registrado previamente por Henríquez \& Bahamonde (1964), para la zona de San Antonio, quienes también informaron que esta presa aumentaba su aparición (66\%) en los estómagos de G. maculatus durante el verano.

Por otro lado, la diversidad fue alta en todas las estaciones del periodo 1996-97, alcanzando su máximo en primavera (Fig. 3), la estación que resultó distinta al compararla con el invierno, debido a la mayor cantidad de especies de peces que consumieron los congrios. En cambio, la dominancia se mantuvo constante, ya que en todas las estaciones, exceptuando otoño, $P$. monodon dominó en la dieta. Resultados similares fueron encontrados por Chong et al. (2000) en la corvina Cilus gilberti (Abbott, 1889) pero con una menor diversidad de presas, por lo que clasificaron a esta especie como un depredador estenofágico. En este contexto, G. chilensis se comportaría como un depredador eurifágico.

Genypterus chilensis se alimenta principalmente de $P$. monodon al igual que $G$. maculatus y $G$. blacodes en Chile (Henríquez \& Bahamonde 1964, Bahamonde \& Zavala 1981), aunque las tres especies viven en profundidades distintas. Esta similitud dietaria indicaría que las tres especies se alimentarían en rangos semejantes de profundidad, y es muy probable que solapen su nicho trófico, aspecto que debería estudiarse para comprender mejor las relaciones ecológicas entre estas tres especies.

\section{Agradecimientos}

Esta investigación fue financiada por la Dirección de Investigación de la Universidad Católica Santísima Concepción, Proyecto DIN-496. Agradecemos también las observaciones y sugerencias al manuscrito de dos revisores anónimos.

\section{Literatura citada}

Avilés S. 1979. Congrio colorado, Genypterus chilensis (Guichenot) Teleostomi, Perciformes, Ophidiidae. En: Estado actual de las principales Pesquerías Nacionales. Bases para una Desarrollo Pesquero. CORFO, IFOP, Santiago, Tomo I, Peces 14, 23 pp.

Bahamonde N \& P Zavala. 1981. Contenidos gástricos de Genypterus maculatus (Tschudi) y Genypterus blacodes (Schneider) capturados en Chile entre $31^{\circ}$ y $37^{\circ} \mathrm{S}$ (Teleostomi, Ophidiidae). Boletin del Museo Nacional de Historia Natural, Chile 38: 53-59.

Chirichigno N. 1974. Clave para Identificar los Peces Marinos del Perú. Informe Instituto del Mar del Perú, Callao 46: 1-109.

Chocair J, F Orellana \& J Serra. 1969. Estudio del género Genypterus (congrios) en aguas chilenas (Pisces: Ophidiidae). Tesis de Biología Marina, Universidad de Chile, Valparaíso, 48 pp.

Chong J, N Cortés \& R Bustos. 2000. Hábitos alimentarios de la corvina Cilus gilberti (Abbott, 1889) (Pisces: Sciaenidae) frente a la costa de Talcahuano. Biología Pesquera 28: 29-35.

George-Nascimento M, V Lobos, C Torrillos \& R Khan. 2004. Species composition of assemblages of Ceratomyxa (Myxozoa), parasites of lings Genypterus (Ophidiidae) in the Southeastern Pacific Ocean: an ecomorphometric approach. Journal of Parasitology 90(6): 1352-1355.

Hammer Ø, DAT Harper \& PD Ryan. 2001. PAST: Paleontological Statistics Software Package for Education and Data Analysis. Palaeontología Electrónica 4: 1-9.

Henríquez G \& N Bahamonde. 1964. Análisis cualitativo y cuantitativo del contenido gástrico del congrio negro (Genypterus maculatus Tschudi) en pescas realizadas entre San Antonio y Constitución (1961-1962). Revista Universitaria 49: 139-158.

Hyslop J. 1980. Stomach contents analysis: A review of methods and their application. Journal of Fish Biology 17: 411-429.

Ludwig JA \& JF Reynolds. 1988. Statistical Ecology, 337 pp. John Wiley \& Sons, Nueva York. 
Mann G. 1954. La vida de los peces en aguas chilenas. Instituto de Investigaciones Veterinarias. Universidad de Chile. 343 pp.

Mitchell SJ. 1984. Feeding of ling Genypterus blacodes (Bloch \& Schneider) from 4 New Zealand offshore fishing grounds. New Zealand Journal of Marine and Freshwater Research 18: 265-274.

Norman JR. 1937. Coast Fishes. Part II. The Patagonian Region. Discovery Report 16: 1-150.

Nyegaard M, A Arkhipkin \& P Brickle. 2004. Variation in the diet of Genypterus blacodes (Ophidiidae) around the Falkland Islands. Journal of Fish Biology 65: 666-682.

Pequeño G. 1989. Peces de Chile. Lista sistemática revisada y comentada. Revista de Biología Marina, Valparaíso 24(2): $1-132$.

Pinkas L, MS Oliphant \& IL Iverson. 1971. Food habits of albacores, bluefin tuna and bonito in California water, California Department of Fish and Game. Fish Bulletin $152,105 \mathrm{pp}$.

Retamal MA. 1981. Catálogo ilustrado de los crustáceos decápodos de Chile. Gayana Zoología 44: 1-110.

Nesis K. 1987. Cephalopods of the world. T.F.H. Publications Inc. New Jersey. 351 pp.
Ruiz VH. 1995. Catálogo de los peces marinos comunes de la Octava Región. Universidad de Concepción. 226 pp.

SERNAPESCA 2004. Anuario Estadístico de Pesca. Servicio Nacional de Pesca. Ministerio de Economía, Fomento y Reconstrucción. Chile. 210 pp.

Tascheri R, J Sateler, J Merino, E Díaz, V Ojeda \& M Montecinos. 2003. Estudio biológico-pesquero de congrio colorado, congrio negro y congrio dorado en la zona centro-sur. Instituto de Fomento Pesquero. Informe Final FIP 2001-15, 300 pp.

Vergara L \& M George-Nascimento. 1982. Contribución al estudio del parasitismo en el congrio colorado, Genypterus chilensis (Guichenot, 1848). Boletín Chileno de Parasitología 37: 9-14.

Vivanco M. 1999. Análisis estadístico multivariable, 234 pp. Editorial Universitaria. Santiago, Chile.

Windell JT. 1968. Food analysis and rate of digestion. En: Ricker WE (ed). Methods for assessment of fish production in freshwaters, IBP Handbook 3: 197-203.

Zar JH. 1984. Biostatistical analysis. Second Edition. Prentice-Hall, Inc., Nueva York.

Recibido el 20 de abril de 2006 y aceptado el 28 de agosto de 2006 